\title{
Local control of hepatocellular carcinoma and colorectal liver metastases after surgical microwave ablation without concomitant hepatectomy
}

\author{
Luís Filipe Abreu de Carvalho $^{1} \cdot$ Bram Logghe $^{1} \cdot \operatorname{Stijn}_{\text {Van Cleven }}{ }^{1} \cdot$ Aude Vanlander $^{1} \cdot$ Suzane Moura Ribeiro ${ }^{2}$. \\ Karen Geboes ${ }^{2} \cdot$ Clarisse Lecluyse $^{3} \cdot$ Peter Smeets $^{3} \cdot$ Helena Degroote $^{4} \cdot$ Hans Van Vlierberghe $^{4} \cdot$ Frederik Berrevoet $^{1}$
}

Received: 25 February 2021 / Accepted: 27 May 2021

(C) The Author(s), under exclusive licence to Springer-Verlag GmbH Germany, part of Springer Nature 2021

\begin{abstract}
Purpose Microwave ablation (MWA) is an accepted technique in the multimodal treatment of hepatocellular carcinoma (HCC) and colorectal liver metastases (CRLM). Study endpoints were to evaluate the local efficacy of surgical MWA in selected patients with oligonodular disease without the combination of liver resection to allow a clear interpretation of the follow-up imaging and compare it to the results on percutaneous MWA available in the literature.

Methods Consecutive MWA-only procedures performed between May 2013 and May 2018 for HCC and CRLM with free-hand ultrasound guidance were identified. MWA systems with $2450 \mathrm{MHz}$ were used. Incomplete ablation (IA) was defined as residual disease within $1 \mathrm{~cm}$ of the ablation site at the first post-ablation imaging and local recurrence (LR) as the presence of disease after at least one tumor-free imaging.

Results A total of 70 tumors in 47 patients were treated with 46 laparoscopic and 1 open procedures. Each patient had no more than 3 tumors, and median size of the lesions was $15 \mathrm{~mm}$ (IQR: 10-22). After a median follow-up of 26 months (IQR: 12-40), IA rate was $8.6 \%$ and LR rate was $29.4 \%$. Multivariable analysis showed that vascular proximity $(\mathrm{OR}=3.4 ; 95 \% \mathrm{CI}=1.26-9.22$; $\mathrm{p}=0.016$ ) was the only significant predictor of the combined outcome IA or LR.

Discussion In the present study, after mostly laparoscopic MWA, LR was higher than the rates available in the literature for percutaneous MWA of HCC but lower than in the limited studies analyzing isolated percutaneous MWA of liver metastases. Future developments may help establish the role of each therapeutic modality per tumor, in order to improve the outcomes.
\end{abstract}

Keywords Liver $\cdot$ Surgery $\cdot$ Laparoscopy $\cdot$ Ablation $\cdot$ HCC $\cdot$ Colorectal liver metastases

Luís Filipe Abreu de Carvalho

luis.abreudecarvalho@uzgent.be

Bram Logghe

bram.logghe@ugent.be

Stijn Van Cleven

stijnvancleven@gmail.com

Aude Vanlander

aude.vanlander@ugent.be

Suzane Moura Ribeiro

suzane.ribeiromoura@uzgent.be

Karen Geboes

karen.geboes@ugent.be

Clarisse Lecluyse

clarisse.lecluyse@uzgent.be

Peter Smeets

peter.smeets@uzgent.be
Helena Degroote

helena.degroote@uzgent.be

Hans Van Vlierberghe

hans.vanvlierberghe@uzgent.be

Frederik Berrevoet

frederik.berrevoet@ugent.be

1 Department of HPB Surgery and Liver Transplantation, Ghent University Hospital, Ghent, Belgium

2 Division of Digestive Oncology, Department of Gastroenterology, Ghent University Hospital, Ghent, Belgium

3 Department of Radiology, Ghent University Hospital, Ghent, Belgium

4 Division of Hepatology, Department of Gastroenterology, Ghent University Hospital, Ghent, Belgium 


\section{Introduction}

Ablation techniques provide an extra tool in the treatment of hepatic tumors by allowing a shorter and less morbid procedure. It is an attractive option for patients in a poor clinical state or with a more reserved oncologic prognosis, which would not warrant a riskier resection. The most frequent indications for liver surgery and thermal ablation are hepatocellular carcinoma (HCC) and colorectal liver metastases (CRLM). Ablations can be combined with liver resections in a parenchyma-preserving approach for CRLM $[1,2]$. Unfortunately, this comes at the cost of a higher local recurrence rate than surgery, which remains the gold standard [3]. Radiofrequency ablation (RFA) is effective in the treatment of HCC although not superior to surgery [4, 5]. Microwave ablation (MWA), with its higher energy, generates higher temperatures in a shorter period of time and seems to provide a better local control than RFA, with less blood driven heat sink effect and permitting to target larger lesions $[1,6]$.

Percutaneous ablations offer a minimally invasive option for the local treatment of hepatic malignancies with low morbidity and possibility of CT guidance to target lesions barely visible with ultrasound [7]. Their therapeutic role in the treatment of very early and early stage HCC has been broadened from patients ineligible for a hepatectomy to an alternative to liver resection until the possibility of being adopted as firstline therapy according to the current European Association for the Study of the Liver clinical practice guidelines [7]. There is less experience with the local efficacy of isolated MWA for CRLM in comparison to HCC [8].

In the quest for minimally invasive treatment options for hepatic malignancies, the choice between hepatectomy and ablation can be made after weighing the morbidity of the procedure against the expected efficacy according to the residual liver function, location of the lesion, and comorbidities of the patient [3-5]. If an ablation is preferred, the choice still has to be made between a CT-guided percutaneous procedure and intraoperative ablation with an open or laparoscopic approach. In order to establish the role of each modality of ablation, their local efficacy should be known and compared.

The evaluation of the results of hepatic ablation is challenging when this is combined with hepatic resections, making it difficult to discern which outcomes precisely derive from thermal ablation. A postoperative hypodensity on imaging can correspond to the expected ablation zone, or it can indicate an ischemic area caused by a neighboring resection. In combined hepatic procedures, it also becomes more complex to differentiate between a local or "de novo" hepatic recurrence. Marginal hyperemia around the ablation zone can be seen in the early phases post-ablation, and this finding can be mistaken for residual tumor. Sequential imaging analysis before and after the ablation is essential. The first cross-sectional imaging after thermal ablation should not show suspect contrast enhancement in the ablation zone and should display enough overlap by having a wider diameter than the original lesion, in order to be considered a complete ablation. The detection of asymmetric contrast enhancement in ulterior imaging or increase in diameter of the ablation zone is considered as suspect for a local recurrence (LR) [9].

Several predictive factors for incomplete ablation (IA) or LR have been proposed in literature such as etiology (HCC versus CRLM), location in the posterosuperior segments (segments $1,4 a, 7,8$ ), tumor size, vascular proximity, superficial location, and requirement of multiple ablations of the same lesion $[1,10]$.

In order to evaluate the efficacy of MWA on the local control of liver tumors and to identify predictive factors for IA and LR, we retrospectively reviewed the patients with HCC and CRLM, treated in Ghent University Hospital with sole surgical MWA without combination with liver resection or any other type of procedure. We compared the results with the available literature on percutaneous MWA.

\section{Material and methods}

\section{Patient selection and collection of data}

In this retrospective single-center study, all consecutive patients who underwent a surgical MWA with curative intent for HCC and CRLM at the Ghent University Hospital between May 2013 and May 2018 were identified, based on the review of clinical files and operation records. Patients with combined procedures were excluded in order to better evaluate the local effects of the ablation, the postoperative course, and the efficacy of the local control throughout follow-up. All patients were discussed at a multidisciplinary oncology meeting where the surgical indication and therapeutic modality were agreed upon. Whenever possible, surgical resection of the tumors was preferred. Patients were selected for ablation in case of cirrhosis with anticipated high-risk hepatectomy for liver failure or in case of extensive surgical or medical history that favored a less invasive approach. The indication for intraoperative ablation was established preoperatively. The study protocol and all the procedures were performed according to the institutional ethical standards of Ghent University Hospital (reference number: B67020173366). The data collected included demographics, details on the surgical procedure and postoperative period, morbidity, mortality, characteristics of the hepatic lesions, and iconography.

\section{Study endpoints}

The primary endpoint of the study was to evaluate the efficacy of MWA as a local treatment for HCC and CRLM by 
calculating the rate of incomplete ablation (IA) and local recurrence (LR) after MWA.

IA of a hepatic tumor was established as the presence of residual tumor within $1 \mathrm{~cm}$ of the ablation site at the first control imaging with computed tomography (CT) or magnetic resonance imaging (MRI) after MWA. LR was defined as tumor recurrence at the site of ablation after at least one imaging without evidence for rest tumor. IA and LR are manifestations of the same conjuncture of residual tumor cells in the ablation zone, albeit chronologically segregated by the interpretation of imaging exams. Therefore, these 2 entities were combined in order to analyze the possible influence of risk factors which can be associated with their occurrence. Recurrence analysis was performed per patient and per tumor.

Secondary endpoints were the assessment of postoperative outcomes such as operation time, morbidity, and hospital stay, as well as the identification of possible predictive factors for IA and LR. The results were compared with the available literature on percutaneous MWA.

\section{Surgical procedure and follow-up}

MWA was performed by two senior surgeons of the Department of Hepatopancreatobiliary Surgery, during laparoscopic or open surgery, under free-hand guidance of non-enhanced ultrasonography. They were responsible for the technical planning of the procedure. Ablations with RFA were performed between 2007 and 2013, but these procedures were excluded from the analysis. Two different MWA probes were used according to the time period. Firstly, AMICA $3.0^{\mathrm{TM}}$ microwave system $(2450 \mathrm{MHz}$, HS Hospital Service, Via Zosimo, Rome, Italy) was used from May 2013 until December 2015 and afterwards, Emprint ${ }^{\mathrm{TM}}$ with thermosphere technology cooled tip antenna $(2450 \mathrm{MHz}$, Medtronic@; Medtronic Parkway, Minneapolis, USA) was used until the end of the study in May 2018. The ablation probe was introduced in the liver under free-hand ultrasonography guidance until the center of the hepatic tumor, after which the ablation was performed to achieve the calculated ablation zone which should include an ablative margin of 5-10 $\mathrm{mm}$. Wattage and time were selected according to the equipment recommendations. Tumor size was assessed based on the preoperative cross-sectional imaging at the point of maximal diameter. In case of a superficial lesion (location at $<5-\mathrm{mm}$ depth from the liver surface) or a lesion located nearby a portal pedicle ( $<5-\mathrm{mm}$ distance), the ablation proceeded with lower power and longer ablation time in order to achieve the desired ablation volume, so that the risk of "popcorn effect" with a burst superficial lesion and peritoneal seeding could be minimized, as well as the risk of biliary thermal injury in case of a neighboring portal pedicle. Pringle maneuver diminishes the vascular flow in the portal pedicles and hence minimizes the "heat-sink" effect in case of a neighboring lesion but also increases the risk of bile duct injury and subsequent bilioma by increasing the heating. Therefore, this strategy was not applied. Vascular proximity was defined as present when a lesion was located at less than $5 \mathrm{~mm}$ of a vascular structure (hepatic vein or portal pedicle). Follow-up occurred according to the supervision of the surgeon, hepatologist, or oncologist in charge, following the existent guidelines. The first contrastenhanced computed tomography (CT) or magnetic resonance imaging (MRI) was planned within 3-6 weeks after the surgery. All available imaging was reassessed by two dedicated abdominal radiologists.

\section{Statistical analysis}

Continuous variables were described with median and interquartile range (IQR), and they were compared using Student's t-test in case of a normal distribution; otherwise, MannWhitney U test was applied. Categorical data were described with counts and percentages. Chi-square test and Fisher's exact test were used to compare categorical variables. Generalized estimating equations (GEE) was used to identify possible prognostic factors associated with LR and IA as a dichotomous combined outcome: tumor presence in a hepatic lesion or not. This methodology was used to account for clustering in the data as some of the patients had more than one lesion and the data was analyzed per lesion. The chosen working correlation structure was "exchangeable." Odds ratio (OR) and $95 \%$ confidence interval (CI) were used to interpret the results. All statistical analyses were performed with IBM® SPSS® statistics 27.0 (2020, Armonk, New York, USA), except for GEE which was performed in open-source R software [11]. A p-value of $<0.05$ was considered statistically significant.

\section{Results}

A total of 128 patients were screened, and 47 patients were included in the study. Sixty-nine patients were excluded because of a combined procedure. There was a combination with liver resections in 58 patients, 5 of whom had an ALPPS procedure (associating liver partition and portal vein ligation for staged hepatectomy); in 10 patients, there was a combination with colorectal resections, 4 of whom with cytoreductive surgery and HIPEC (hyperthermic intraperitoneal chemotherapy). One patient had a concomitant esophagectomy. Three additional patients were excluded because MWA was performed despite clear systemic progression of the disease, because of reasons specified during the multidisciplinary tumor board meeting. The baseline characteristics of the study population are shown in Table 1.

CRLM patients had more frequently a previous laparotomy [13 $(77 \%)$ vs $2(7 \%), \mathrm{p}<0.001]$ and had more often multiple lesions [11 (65\%) vs $5(17 \%), \mathrm{p}=0.002]$ which could explain the longer operation time [155 $\min (118-223)$ vs 110 (77- 
Table 1 Clinical characteristics

\begin{tabular}{|c|c|c|c|c|}
\hline Characteristic & $\operatorname{CRLM}(\mathrm{n}=17)$ & $\mathrm{HCC}(\mathrm{n}=30)$ & Total $(n=47)$ & $\mathrm{p}$ \\
\hline$\%$ cases & 36 & 64 & 100 & NA \\
\hline \multicolumn{5}{|l|}{ Age } \\
\hline Median, yr (IQR) & $62(52-74)$ & $65(58-71)$ & $64(52-72)$ & 0.760 \\
\hline \multicolumn{5}{|l|}{ Sex, n $(\%)$} \\
\hline Female & $2(11.8)$ & $8(26.7)$ & $10(21.3)$ & \multirow[t]{2}{*}{0.289} \\
\hline Male & $15(88.2)$ & $22(73.3)$ & $37(78.7)$ & \\
\hline \multicolumn{5}{|l|}{ BMI } \\
\hline Median, kg/m2 (IQR) & $24(22-30)$ & $27(24-31)$ & $26(23-30)$ & 0.295 \\
\hline \multicolumn{5}{|l|}{ Child-Pugh score, n (\%) } \\
\hline A & NA & $23(77)$ & NA & \multirow[t]{2}{*}{ NA } \\
\hline $\mathrm{B}$ & NA & $7(23)$ & NA & \\
\hline \multicolumn{5}{|l|}{ MELD-score } \\
\hline Median, (range) & NA & $10(8-12)$ & NA & NA \\
\hline \multicolumn{5}{|c|}{ Location of primary CRC, $\mathrm{n}(\%)$} \\
\hline Right-sided & $4(24)$ & NA & NA & \multirow[t]{3}{*}{ NA } \\
\hline Left-sided & $8(47)$ & NA & NA & \\
\hline Rectum & $5(29)$ & NA & NA & \\
\hline \multicolumn{5}{|l|}{ Operation time } \\
\hline Median, min (range) & $155(118-223)$ & $110(77-141)$ & $120(95-160)$ & 0.001 \\
\hline Previous laparotomy, n (\%) & $13(77)$ & $2(7)$ & $15(32)$ & $<0.001$ \\
\hline \multicolumn{5}{|l|}{ Approach, n (\%) } \\
\hline Open & $1(5.9)$ & $0(0)$ & $1(2.1)$ & \multirow[t]{2}{*}{0.362} \\
\hline Laparoscopic & $16(94.1)$ & $30(100)$ & $46(97.9)$ & \\
\hline \multicolumn{5}{|c|}{ Number of hepatic lesions, n (\%) } \\
\hline 1 & $6(35)$ & $25(83)$ & $31(66)$ & \multirow[t]{3}{*}{0.002} \\
\hline 2 & $8(47)$ & $4(13)$ & $12(25.5)$ & \\
\hline 3 & $3(18)$ & $1(4)$ & $4(8.5)$ & \\
\hline \multicolumn{5}{|c|}{ Clavien-Dindo classification, n (\%) } \\
\hline $\mathrm{I}-\mathrm{II}$ & $2(11.8)$ & $1(3.3)$ & $3(6.4)$ & \multirow[t]{2}{*}{0.792} \\
\hline III-IV & $1(5.9)$ & $2(6.7)$ & $3(6.4)$ & \\
\hline \multicolumn{5}{|l|}{ Hospital stay } \\
\hline Median, days (range) & $3(3-5)$ & $3(2-4)$ & $3(2-4)$ & 0.290 \\
\hline
\end{tabular}

$N A$ not applicable, $C R C$ colorectal cancer, $I Q R$ interquartile range

141), $\mathrm{p}=0.001]$ due to the adhesiolysis and extra ablations required. With exception of these 3 mentioned features, there were no major differences between the CRLM and HCC subpopulations (Table 1). Only 1 patient underwent an open procedure despite previous laparotomy in 13 (77\%) patients with CRLM and 2 (7\%) patients with HCC.

Severe morbidity was observed in 3 patients (ClavienDindo III-IV, 6.4\%) with one case of bleeding, one case of colonic perforation, and one late case of bile duct dilation after ablation of a central lesion in segment 8 , which eventually required the resection of the hepatic anterior sector, 6 months after the ablation because of a bile leak and dilation of the bile ducts in this sector. There was no perioperative mortality.

When we analyze the results per patient (Table 2), the most frequent found sites of first recurrence were "remote hepatic recurrence only" (11 cases, 23.9\%) and "local recurrence" (10 cases, 21.7\%). Median follow-up was 26 months (IQR: 12 40). The percentage of patients with no recurrence was higher in the HCC group in comparison to the CRLM group [13 $(43.3 \%)$ vs $4(25 \%), \mathrm{p}=0.352]$. The median time to local recurrence was 6 months shorter in the patients with CRLM [6 (2.5-9)] in comparison to the patients with HCC [12 (7-13)] $(\mathrm{p}=0.015)$ (Table 2).

Six out of 30 patients in the HCC cohort (20\%) had a liver transplantation, and in $2(6.7 \%)$ of these patients, although imaging was not suspect for a local recurrence, pathology report of the explanted liver still showed residual tumor in the ablation site.

A total of 70 hepatic lesions were treated (Table 3). Incomplete ablation rate was $8.6 \%$, and local recurrence rate was $29.4 \%$ on the analysis per tumor, without a significant difference between CRLM and HCC (Table 3). 
Table 2 Per patient analysis

\begin{tabular}{lllll}
\hline Characteristic & CRLM $(\mathrm{n}=17)$ & HCC $(\mathrm{n}=30)$ & Total $(\mathrm{n}=47)$ & $\mathrm{p}$ \\
\hline First site of recurrence, $\mathrm{n}(\%)$ & & & & \\
No recurrence & $4(25)$ & $13(43.3)$ & $17(37)$ & 0.352 \\
Incomplete ablation & $2(12.5)$ & $3(10)$ & $5(10.9)$ & $10(21.7)$ \\
Local recurrence & $3(18.8)$ & $7(23.3)$ & $11(23.9)$ & \\
Remote hepatic recurrence only & $5(31.3)$ & $6(20)$ & $2(4.3)$ & \\
Intra and extra-hepatic recurrence & $2(12.5)$ & $0(0)$ & $1(2.2)$ & \\
Extra-hepatic recurrence only & $0(0)$ & $1(3.3)$ & $26(12-40)$ & 0.689 \\
Follow-up & & $23(10-41)$ & & \\
Median, months (IQR) & $26(16-40)$ & $12(7-13)$ & $8(4.5-12)$ & 0.015 \\
Time to local recurrence & $6(2.5-9)$ & & & \\
Median, months (IQR) & & & & \\
\hline
\end{tabular}

$I Q R$ interquartile range

Multivariable analysis based on GEE showed that vascular proximity $(\mathrm{OR}=3.4 ; 95 \% \mathrm{CI}=1.26-9.22 ; \mathrm{p}=0.016)$ was a significant predictor of the combined outcome IA or LR recurrence, while superficial location of the tumor had a protective effect $(\mathrm{OR}=0.32 ; 95 \% \mathrm{CI}=0.11-0.96 ; \mathrm{p}=0.041)$. Also, the size of the tumor $(>20 \mathrm{~mm}$ ) showed a tendency to be associated with higher odds of IA or LR with a marginal significant $\mathrm{p}$-value $(\mathrm{OR}=3.43,95 \% \mathrm{CI}=0.88,13.41, \mathrm{p}=0.076)$ (Table 4).

Etiology, tumor location in the posterosuperior segments, and necessity of multiple placement of the ablation probe to treat the same lesion did not have a predictive value for the combined outcome LR or IA (Table 4).

\section{Discussion}

Surgical possibilities in minimally invasive liver resections are increasing not only with parenchyma preserving approaches for CRLM but also with the proposal of liver resections for HCC in Child B liver cirrhosis [12-14]. As both the local efficacy of MWA and the technical possibilities of liver resection increase, while the morbidity of surgery diminishes, this separation line may blur. In case there are no associated liver resections planned, the choice between operative ablation with laparoscopy and a percutaneous procedure should be posed by considering their success rates and morbidity.

In this analysis, the feasibility of surgical MWA of hepatic tumors is corroborated as well as the applicability of laparoscopy as a safe option after previous open abdominal surgery. However, the reported rates of IA and LR are higher than expected (Tables 2 and 3 ).

Our observed IA rate was $8.6 \%$, and LR rate was $29.4 \%$ for the total of 70 lesions, which is higher than the general results available in literature (Table 3). In the review by Gang Yang et al. with inclusion of studies comparing MWA with liver resection for the treatment of HCC and liver metastases, the incidence of local tumor recurrence in the MWA group was $16.7 \%$ [8]. Operative ablations and percutaneous ablations with $\mathrm{CT}$ and ultrasound guidance were included in the review, and it was not always mentioned whether the analysis was per tumor or per patient, warranting a closer analysis to discern

Table 3 Per tumor analysis

\begin{tabular}{lllll}
\hline Characteristic & CRLM $(\mathrm{n}=31)$ & HCC $(\mathrm{n}=39)$ & Total $(\mathrm{n}=70)$ & $\mathrm{P}$ \\
\hline $\begin{array}{l}\text { \% cases } \\
\text { Tumor size }\end{array}$ & 44.3 & 55.7 & 100 & NA \\
Median, mm (IQR) & $13(10-20)$ & $18(10-20)$ & $15(10-22)$ & 0.602 \\
Vascular proximity, n (\%) & $7(24.1)$ & $5(12.8)$ & $12(17.6)$ & 0.226 \\
Superficial location, n (\%) & $14(48.3)$ & $16(41)$ & $30(44.1)$ & 0.552 \\
Tumor in PS segments, $\mathrm{n}(\%)$ & $19(61.3)$ & $26(66.7)$ & $45(64.3)$ & 0.641 \\
Multiple ablation, $\mathrm{n}(\%)$ & $9(29)$ & $15(39.5)$ & $24(34.8)$ & 0.365 \\
Incomplete ablation, $\mathrm{n}(\%)$ & $3(9.7)$ & $3(7.7)$ & $6(8.6)$ & 1 \\
Local recurrence, $\mathrm{n}(\%)$ & $9(29)$ & $11(29.7)$ & $20(29.4)$ & 0.950 \\
\hline
\end{tabular}

PS posterosuperior segments ( $\mathrm{Sg} 1,4 \mathrm{a}, 7,8), N A$ not applicable, IQR interquartile range 
Table 4 Analysis of predictive factors for incomplete ablation and local recurrence by generalized estimating equations

\begin{tabular}{lllll}
\hline & Univariable OR (95\% CI) & Univariable p-value & Multivariable OR (95\% CI) & Multivariable p-value \\
\hline Etiology (HCC vs CRLM) & $0.9(0.26-3.12)$ & 0.864 & $0.86(0.23-3.27)$ & 0.83 \\
Tumor in PS segments & $0.69(0.26-1.83)$ & 0.459 & $1.31(0.55-3.15)$ & 0.544 \\
Tumor size $\geq 20 \mathrm{~mm}$ & $2.13(0.64-7.09)$ & 0.216 & $3.43(0.88-13.41)$ & 0.076 \\
Vascular proximity & $2.42(0.91-6.42)$ & 0.076 & $3.4(1.26-9.22)$ & 0.016 \\
Superficial location & $0.39(0.14-1.09)$ & 0.073 & $0.32(0.11-0.96)$ & 0.041 \\
Multiple ablation & $0.83(0.36-1.9)$ & 0.664 & $0.66(0.29-1.48)$ & 0.316 \\
\hline
\end{tabular}

No. of clusters 46

No. of observations 67

PS posterosuperior segments (Sg 1, 4a, 7, 8)

possible differences [8]. In the study from Takami et al., intraoperative MWA of HCC was performed by laparoscopy, laparotomy, or thoracotomy achieving a local recurrence rate at 5 years as low as $5.9 \%$ for a mean tumor size of $26.9 \mathrm{~mm}$ and after a mean follow-up of 45.9 months [15]. Baker et al. reported a local recurrence rate of $8.5 \%$ after a shorter followup of 10.9 months, and most of the procedures were performed in a laparoscopic fashion. These two reports show very favorable local control rates after intraoperative ablation to which our series with mostly laparoscopic MWA compares inferiorly. In contrast to these results, a retrospective study with also laparoscopic MWA, comprising 674 patients with $\mathrm{HCC}$, showed a local tumor progression rate at 1 and 3 years of $28.6 \%$ and $40.9 \%$, respectively, to which our results compare favorably [16]. Laparoscopic ablations are technically more demanding, and that could explain a worse local control in this study by Cillo et al. [16]. In opposition to ablation in open surgery, there is no free movement of the ablation antenna after defining the passing point of the antenna through the abdominal wall. In our experience, the establishment of the trajectory line for the ablation during laparoscopy can be very challenging and depends on factors such as pneumoperitoneum, the angle of approach of the hepatic lesion and relation to the rib cage, possible need for hepatic mobilization, and coordination between laparoscopic ultrasound and calculation of punction trajectory in the liver. These factors may contribute to an altered approach of the liver anatomy and weaken the success of ablation by laparoscopy. Guidance systems and planning software may increase the accuracy of laparoscopic ablations by helping establish the optimal trajectory of the ablation probe and target the lesion more efficiently than with free-hand ultrasound guidance [17].

Studies concerning percutaneous MWA of HCC show a local recurrence rate between 9.9 and $19 \%$ using CT or ultrasound guidance [18-21]. These results are better than our local recurrence rate per tumor of $29.7 \%$ in the present series. Percutaneous ablations show possible advantages in patients with a cirrhotic liver and/or concomitant comorbidities in comparison to an open or laparoscopic procedure by its low invasiveness and low morbidity, and they offer the possibility of CT guidance to target lesions barely visible with ultrasound [7]. Direct percutaneous approach of an hepatic lesion can be an advantage after extensive liver surgery or in cirrhotic livers, avoiding cumbersome liver mobilization or the interruption of collateral circulation. On the other hand, recent studies suggest a possible wider role for liver resection in patients with HCC and Child B liver cirrhosis [14]. The experience illustrated in this retrospective cohort derives from a period when there was no rigid pre-defined therapeutic pathway and in which the gold standard of liver resection or at least laparoscopic exploration was probably pursued in a more resolute fashion. Based on recent developments, it seems reasonable to apply percutaneous CT-guided ablation for the treatment of small HCC tumors, within Milan criteria as bridge to liver transplantation [7]. Certain circumstances are not ideal for a percutaneous approach, and the possibility of offering a laparoscopic exploration remains a useful therapeutic tool like when the tumor is located near the gallbladder, viscera, or diaphragm [19]. Superficial tumors are usually to be resected, but the satisfactory results in the present cohort with ablation of superficial tumors could be an extra argument to use MWA in selected cases. We hypothesized that superficial tumor location would be a risk factor for IA or LR after MWA due to the limitations in the use of high energy to prevent a "popcorn effect," but it rather proved to be a protective factor against incomplete local control (Table 4). Apparently, an algorithm with lower energy and longer ablation time also achieved a satisfactory local control.

The two most frequent sites of first recurrence in the total cohort of this trial were "remote hepatic recurrence only" (23.9\%) and "local recurrence" (21.7\%) (Table 2). There is evidence that ablation techniques may promote tumor growth distant from the primary ablation site through the expression of cytokines and growth factors promoted by the 
periablational inflammatory response [22]. A meta-analysis comparing RFA with surgical resection for CRLM showed an increased rate of local and "de novo" intrahepatic recurrences in the RFA group [3]. A retrospective study involving imaging review in 59 patients reported a higher growth rate of new HCC's after RFA in comparison to the expected rate in untreated HCC's [23]. Faster MWA protocols with higher temperatures may reduce the expression of inflammatory markers and limit their tumorigenic effects in comparison to slower RFA or MWA procedures with lower temperatures. Application algorithms can become even more complex if one should take into account not only how to obtain the anticipated ablation zone, but also how to limit their undesired distant effects [22]. It would be interesting to evaluate whether patients treated for superficial lesions with lower energy and longer ablation time MWA would develop more intrahepatic recurrences, but this limited and heterogeneous cohort did not allow multiple analysis.

The treatment of liver metastases with isolated percutaneous ablations is less frequently described in the literature and shows poorer results concerning local tumor control. The group from Oxford performed $126 \mathrm{CT}$-guided percutaneous ablations in 87 patients with CRLM. With a median tumor size of $34 \mathrm{~mm}$, the ablation site recurrence rate was $34 \%$ [24]. Alexander et al. analyzed the results of MWA in the treatment of 64 patients with solitary hepatic malignancies (HCC, CRLM, other metastases), mostly treated with percutaneous CT-guided ablation. With a median tumor size of between 8 and $12 \mathrm{~mm}$, the 1-year local recurrence rate was $39.8 \%$ for HCC and $45.7 \%$ for CRLM [25]. These findings underline the role of biological factors in the local tumor control. Our series compares favorably with these 2 studies.

Technical skills, local settings, and local expertise remain important biases, difficult to homogenize between studies. In the current series, MWA was used in a selected population with 3 or less hepatic nodules and size inferior to $3 \mathrm{~cm}$, with up to date MWA generators as a bail-out strategy for tumors for which resection was deemed cumbersome or too extensive in relation to the liver function or comorbidities of the patient. The initial period of RFA and MWA application in our department was excluded to minimize the effect of learning curve. The application of laparoscopy in the context of CRLM seems justified for an abdominal exploration and ultrasound evaluation of the liver, which can lead to the identification of new lesions or lesions thought to be missing after chemotherapy, clearer identification of the anatomical relations of the metastases, and possible documentation of peritoneal metastases. All these findings may lead to an intraoperative modification of the therapeutic strategy from liver resection to R1 vascular resection or ablation in a laparoscopic or open fashion [26]. In the context of hepatic recurrence after extensive liver surgery for CRLM, a percutaneous ablation may be preferred depending on the size and location of the lesion, as laparoscopy may not be feasible and an open approach may not justify the possible morbidity which would delay the resume of eventual chemotherapy.

Vascular proximity is a known risk factor for local recurrence which was confirmed in our study (Table 4) [6]. Sequential ablation with repositioning of the probes is usually associated with a less predictable result in comparison with systems where simultaneous multiple applicators are possible [1]. Repositioning of the probe is also associated with bleeding and tumor seeding [27]. After initial ablation, it can be difficult to reposition the antenna with ultrasound guidance and efficiently target the tumor with the postablational artefacts. In the present analysis, $34.8 \%$ of the tumors were treated with multiple ablation by repositioning of the probe, but this was not a risk factor for the combined outcome IA or LR. Given the retrospective character of this study, the reason for sequential ablation was not always evident in the operation report, but an unsatisfying result on ultrasound after the first ablation could be assumed. CT-guided percutaneous MWA with intermediate scans to control the position of the probe and the result of the ablation may offer an advantage. A report on CT-guided stereotactic navigation MWA showed a good accuracy, and almost no repositioning of the antenna was required [27].

After a median follow-up of 26 months (12-40), there was a significant shorter median time to local recurrence in the patients with CRLM (6 months, 2.5-9) contrasting with the HCC patients (12 months, 7-13) $(\mathrm{p}=0.015)$ (Table 2). There were no local recurrences after 1-year follow-up in the patients with CRLM, whereas this was still observed in the HCC group. This could aid to determine the strategy to follow the patients for the evaluation of local control in the liver. In CRLM, if findings are not particularly suspicious upon evaluation after 1 year, it is possibly safe to have a watch and wait strategy, based on these results which show an early local recurrence during follow-up. In HCC we should remain alert for local recurrence until later on. The fact that imaging techniques are not perfect for the detection of tumor at the ablation site is illustrated in this analysis by the diagnosis of ablation site tumor, in 2 of the 6 patients who underwent a liver transplantation for HCC, after unsuspicious imaging [9].

Limitations of this study are its retrospective character and its heterogeneity due to the use of 2 different MWA systems and the joint analysis of CRLM and HCC. Because of the small sample size, the applicability of subgroup analysis was limited. On the other hand, this study tries to translate the reality of MWA application in surgical departments, as being mostly applied for local control of selected cases of HCC and CRLM, while surgery remains the mainstay of treatment. In order to better evaluate the local outcomes of MWA, we selected MWA-only cases, without combination with liver resection or other surgeries. 
Future perspectives in the field of local management of hepatic tumors bring along many technologic developments and interested parties. The challenge will be to establish a therapeutic algorithm with all professionals and tools available, in order to achieve the best treatment possible. The role of the surgeon, in an era where many of these procedures can be performed percutaneously using ultrasonography or CT guidance, is yet to be clarified. The contribution of aiding software systems, 3D imaging reconstruction, fusion imaging, and augmented reality are important developments which may contribute to the improvement of the efficacy of the procedure, allowing a better planning and more accurate guidance for puncture to obtain the desired trajectory and ablation zone $[17,28]$.

\section{Conclusion}

MWA is a powerful tool in the armamentarium for the local treatment of hepatic malignancies. The LR in this study with mostly laparoscopic MWA was higher than the rates available in the literature for percutaneous MWA of HCC but lower than in the limited studies analyzing isolated percutaneous MWA of CRLM. Future developments may help address this matter and clarify the role of each tool in the multimodal therapeutic algorithm per tumor, in order to improve the outcomes.

Acknowledgements The statistical analysis was performed with the assistance of the Department of Biostatistics of Ghent University Hospital.

Availability of data and material Data is available

Code availability Not applicable

Author contribution All authors contributed to the study conception and design. Material preparation, data collection, and analysis were performed by Luís Filipe Abreu de Carvalho, Bram Logghe, Clarisse Lecluyse, and Peter Smeets. The first draft of the manuscript was written by Luís Filipe Abreu de Carvalho, and all authors commented on previous versions of the manuscript. All authors read and approved the final manuscript.

\section{Declarations}

Ethics approval The study protocol and all the procedures were performed according to the institutional ethical standards of Ghent University Hospital (reference number: B67020173366).

Consent to participate Not applicable

Consent for publication Not applicable

Competing interests The authors declare no competing interests.

\section{References}

1. Wells SA, Hinshaw JL, Lubner MG, Ziemlewicz TJ, Brace CL, Lee FT Jr (2015) Liver ablation: best practice. Radiol Clin North Am. 53:933-971. https://doi.org/10.1016/j.rcl.2015.05.012

2. Xourafas D, Pawlik TM, Ejaz A, Dillhoff M, Abdel-Misih S, Tsung A et al (2019) Impact of concomitant ablation on the perioperative outcomes of patients with colorectal liver metastases undergoing hepatectomy: a propensity score matched nationwide analysis. HPB (Oxford) 21:1079-1086. https://doi.org/10.1016/j.hpb.2018. 12.010

3. van Amerongen MJ, Jenniskens SFM, van den Boezem PB, Fütterer JJ, de Wilt JHW (2017) Radiofrequency ablation compared to surgical resection for curative treatment of patients with colorectal liver metastases - a meta-analysis. HPB (Oxford) 19: 749-756. https://doi.org/10.1016/j.hpb.2017.05.011

4. Ng KKC, Chok KSH, Chan ACY, Cheung TT, Wong TCL, Fung JYY, Yuen J, Poon RTP, Fan ST, Lo CM (2017) Randomized clinical trial of hepatic resection versus radiofrequency ablation for early-stage hepatocellular carcinoma. Br J Surg. 104:17751784. https://doi.org/10.1002/bjs. 10677

5. Desiderio J, Trastulli S, Pasquale R, Cavaliere D, Cirocchi R, Boselli C, Noya G, Parisi A (2013) Could radiofrequency ablation replace liver resection for small hepatocellular carcinoma in patients with compensated cirrhosis? A 5-year follow-up. Langenbecks Arch Surg. 398:55-62. https://doi.org/10.1007/ s00423-012-1029-2

6. Izzo F, Granata V, Grassi R, Fusco R, Palaia R, Delrio P, Carrafiello G, Azoulay D, Petrillo A, Curley SA (2019) Radiofrequency ablation and microwave ablation in liver tumors: an update. Oncologist. 24:e990-e1005. https://doi.org/10.1634/ theoncologist.2018-0337

7. European Association for the Study of the Liver (2018) EASL Clinical Practice Guidelines: management of hepatocellular carcinoma. J Hepatol. 69:182-236. https://doi.org/10.1016/j.jhep.2018. 03.019

8. Yang G, Xiong Y, Sun J, Wang G, Li W, Tang T, Li J (2020) The efficacy of microwave ablation versus liver resection in the treatment of hepatocellular carcinoma and liver metastases: a systematic review and meta-analysis. Int J Surg. 77:85-93. https://doi.org/10. 1016/j.ijsu.2020.03.006

9. Patel N, King AJ, Breen DJ (2017) Imaging appearances at followup after image-guided solid-organ abdominal tumour ablation. Clin Radiol. 72:680-690. https://doi.org/10.1016/j.crad.2017.01.014

10. Zaidi N, Okoh A, Yigitbas H, Yazici P, Ali N, Berber E (2016) Laparoscopic microwave thermosphere ablation of malignant liver tumors: an analysis of 53 cases. J Surg Oncol. 113:130-134. https:// doi.org/10.1002/jso. 24127

11. R Core Team (2020). R: A language and environment for statistical computing. Foundation for Statistical Computing, Vienna, Austria. URL https://www.R-project.org/

12. Ferrero A, Lo Tesoriere R, Russolillo N (2019) Ultrasound liver map technique for laparoscopic liver resections. World J Surg. 43: 2607-2611. https://doi.org/10.1007/s00268-019-05046-3

13. Aghayan DL, Fretland ÅA, Kazaryan AM, Sahakyan MA, Dagenborg VJ, Edwin B et al (2019) Laparoscopic versus open liver resection in the posterosuperior segments: a sub-group analysis from the OSLO-COMET randomized controlled trial. HPB (Oxford) 21:1485-1490. https://doi.org/10.1016/j.hpb.2019.03. 358

14. Beard RE, Wang Y, Khan S, Marsh JW, Tsung A, Geller DA (2018) Laparoscopic liver resection for hepatocellular carcinoma in early and advanced cirrhosis. HPB (Oxford) 20:521-529. https://doi.org/10.1016/j.hpb.2017.11.011 
15. Takami Y, Ryu T, Wada Y, Saitsu H (2013) Evaluation of intraoperative microwave coagulo-necrotic therapy $(\mathrm{MCN})$ for hepatocellular carcinoma: a single center experience of 719 consecutive cases. J Hepatobiliary Pancreat Sci. 20:332-341. https://doi.org/ 10.1007/s00534-012-0527-5

16. Cillo U, Bertacco A, Fasolo E, Carandina R, Vitale A, Zanus G, Gringeri E, D'Amico F, Bassi D, Neri D, Dadduzio V, Farinati F, Aliberti C (2019) Videolaparoscopic microwave ablation in patients with HCC at a European high-volume center: Results of 815 procedures. J Surg Oncol. 120:956-965. https://doi.org/10. $1002 /$ jso. 25651

17. Berber E (2015) The first clinical application of planning software for laparoscopic microwave thermosphere ablation of malignant liver tumours. HPB (Oxford). 17:632-636. https://doi.org/10. 1111/hpb.12423

18. Gaia S, Ciruolo M, Ribaldone DG, Rolle E, Migliore E, Mosso E, Vola S, Risso A, Fagoonee S, Saracco GM, Carucci P (2021) Higher efficiency of percutaneous microwave (MWA) than radiofrequency ablation (RFA) in achieving complete response in cirrhotic patients with early hepatocellular carcinoma. Curr Oncol. 28: 1034-1044. https://doi.org/10.3390/curroncol28020101

19. McDevitt JL, Collard MD, Murphy RP, Sutphin PD, Yopp AC, Singal AG et al (2020) Comparison of radiofrequency and microwave ablation and identification of risk factors for primary treatment failure and local progression. Clin Imaging. 67:146-151. https://doi.org/10.1016/j.clinimag.2020.06.014

20. Xu J, Zhao Y (2015) Comparison of percutaneous microwave ablation and laparoscopic resection in the prognosis of liver cancer. Int J Clin Exp Pathol. 8:11665-11669

21. Zhang EL, Yang F, Wu ZB, Yue CS, He TY, Li KY, Xiao ZY, Xiong M, Chen XP, Huang ZY (2016) Therapeutic efficacy of percutaneous microwave coagulation versus liver resection for single hepatocellular carcinoma $\leq 3 \mathrm{~cm}$ with Child-Pugh A cirrhosis. Eur J Surg Oncol. 42:690-697. https://doi.org/10.1016/j.ejso.2016. 02.251

22. Velez E, Goldberg SN, Kumar G, Wang Y, Gourevitch S, Sosna J, Moon T, Brace CL, Ahmed M (2016) Hepatic thermal ablation: effect of device and heating parameters on local tissue reactions and distant tumor growth. Radiology. 281:782-792. https://doi. org/10.1148/radiol.2016152241

23. Park Y, Choi D, Lim HK, Rhim H, Kim YS, Kim SH, Lee WJ (2008) Growth rate of new hepatocellular carcinoma after percutaneous radiofrequency ablation: evaluation with multiphase $\mathrm{CT}$. AJR Am J Roentgenol. 191:215-220. https://doi.org/10.2214/ AJR.07.3297

24. Urbonas T, Anderson EM, Gordon-Weeks AN, Kabir SI, Soonawalla Z, Silva MA, Gleeson FV, Reddy S (2019) Factors predicting ablation site recurrence following percutaneous microwave ablation of colorectal hepatic metastases. HPB (Oxford). 21: 1175-1184. https://doi.org/10.1016/j.hpb.2019.01.007

25. Alexander ES, Wolf FJ, Machan JT, Charpentier KP, Beland MD, Iannuccilli JD, Haas RH, Dupuy DE (2015) Microwave ablation of focal hepatic malignancies regardless of size: a 9-year retrospective study of 64 patients. Eur J Radiol. 84:1083-1090. https://doi.org/ 10.1016/j.ejrad.2015.02.027

26. Ferrero A, Langella S, Giuliante F, Viganò L, Vellone M, Zimmitti G, Ardito F, Nuzzo G, Capussotti L (2013) Intraoperative liver ultrasound still affects surgical strategy for patients with colorectal metastases in the modern era. World J Surg. 37:2655-2663. https:// doi.org/10.1007/s00268-013-2183-6

27. Engstrand J, Toporek G, Harbut P, Jonas E, Nilsson H, Freedman J (2017) Stereotactic CT-guided percutaneous microwave ablation of liver tumors with the use of high-frequency jet ventilation: an accuracy and procedural safety study. AJR Am J Roentgenol. 208: 193-200. https://doi.org/10.2214/AJR.15.15803

28. Park BJ, Hunt SJ, Nadolski GJ, Gade TP (2020) Augmented reality improves procedural efficiency and reduces radiation dose for CTguided lesion targeting: a phantom study using HoloLens 2. Sci Rep. 10:18620. https://doi.org/10.1038/s41598-020-75676-4

Publisher's note Springer Nature remains neutral with regard to jurisdictional claims in published maps and institutional affiliations. 